\title{
ON SPACES IN WHICH THE THREE MAIN KINDS OF COMPACTNESS ARE EQUIVALENT
}

\author{
Woo ChORL Hong
}

\begin{abstract}
In this paper, we introduce a new property $(*)$ of a topological space and prove that if $X$ satisfies one of the following conditions (1) and (2), then compactness, countable compactness and sequential compactness are equivalent in $X$;

(1) Each countably compact subspace of $X$ with $(*)$ is a sequential or AP space.

(2) $X$ is a sequential or AP space with $(*)$.
\end{abstract}

\section{Introduction}

All spaces under consideration are assumed to be Hausdorff. Our terminology is standard and follows [2], [6], and [18].

Let $X$ be a topological space. For each subset $A$ of $X$, we use $\bar{A}$ for the closure of $A$ in $X$. Let $\mathbb{N}$ denote the set of all natural numbers and $\left(x_{n} \mid n \in \mathbb{N}\right)$ (briefly $\left(x_{n}\right)$ ) a sequence of points of a set.

The following functions $[\cdot]_{\text {seq }}$ and $[\cdot]_{A P}$ from the power set $\mathcal{P}(X)$ of $X$ into $\mathcal{P}(X)$ itself defined by for each subset $A$ of $X$,

$$
\begin{aligned}
{[A]_{\text {seq }}=\{x \in X:} & \left(x_{n}\right) \text { converges to } x \text { in } X \text { for some sequence }\left(x_{n}\right) \text { of } \\
& \text { points of } A\}
\end{aligned}
$$

and

$$
[A]_{A P}=A \cup\{x \in \bar{A}-A: \bar{F}=F \cup\{x\} \text { for some subset } F \text { of } A\}
$$

are called the sequential closure operator on $X([2])$ and the AP closure operator on $X([16])$, respectively.

It is well-known that the sequential closure operator $[\cdot]_{\text {seq }}$ and the AP closure operator $[\cdot]_{A P}$ satisfy the Kuratowski topological closure axioms except for

Received January 9, 2008; Revised October 13, 2008.

2000 Mathematics Subject Classification. 54A20, 54B05, 54D30, 54D55, 54E25.

Key words and phrases. Fréchet-Urysohn, sequential, AP, WAP, countable tightness, weakly discretely generated, compact, countably compact, sequentially compact and property $(*)$.

This paper was supported for two years by Pusan National University Research Grant. 
idempotency in general. We see easily that for each subset $A$ of $X$,

$$
A \subset[A]_{\text {seq }} \subset[A]_{A P} \subset \bar{A} \text {. }
$$

Let us recall some properties of a space $X$ which are generalizations of the first countability.

(1) Fréchet-Urysohn ([2]) (also called Fréchet ([7]) or closure sequential ([18])): for each subset $A$ of $X,[A]_{\text {seq }}=\bar{A}$.

(2) sequential ([7]): for each subset $A$ of $X$ which is not closed in $X$, $[A]_{\text {seq }}-A \neq \emptyset$.

(3) AP (standing for Approximation by Points) ([4]) (also called Whyburn ([14])): for each subset $A$ of $X,[A]_{A P}=\bar{A}$.

(4) WAP (standing for Weak Approximation by Points) ([4]) (also called weakly Whyburn ([13])): for each subset $A$ of $X$ which is not closed in $X$, $[A]_{A P}-A \neq \emptyset$.

It is well-known that every first countable space is Fréchet-Urysohn, every Fréchet-Urysohn space is sequential as well as AP, every sequential space is WAP and every AP space is WAP. But, the converses need not be true in general. Moreover, being sequential and being AP are independent and every sequential and AP space is Fréchet-Urysohn. See [2], [3], [4], [7], [8], [9], [10], [11], [13], [14], [16], [17], [18].

The following theorem is a compilation of known facts about these concepts. We will use them to prove our results.

Theorem 1.1. (1) Every Fréchet-Urysohn space is hereditarily Fréchet-Urysohn ([7, Proposition 2.1]).

(2) Every AP space is hereditarily AP ([16, Proposition 2.1(1)]).

(3) Every open or closed subspace of a sequential space is sequential ([7, Proposition 1.9]).

(4) Every countably compact and AP space is Fréchet-Urysohn ([16, Theorem $2.2])$

(5) Every countably compact subspace of a sequential space is closed ([7, Proposition 5.4]).

(6) Every countably compact and WAP space is sequentially compact ([3, Proposition 3] and [10, Corollary 2.8]).

The main purpose of this paper is to investigate the problem of when the three main kinds of compactness: compactness, countable compactness and sequential compactness are equivalent. We introduce a new property $(*)$ of a topological space which is weaker than compactness as well as second countability and show that all paracompact and sequential spaces and all paracompact, weakly discretely generated and AP spaces satisfy $(*)$. We prove that if each countably compact subspace of a space $X$ with $(*)$ is a sequential or AP space, then the three main kinds of compactness are equivalent in $X$; that is, for each subspace $S$ of $X$, the following statements are equivalent: (1) $S$ is compact, (2) $S$ is countably compact and (3) $S$ is sequentially compact. And we have 
that if $X$ is a sequential or AP space with $(*)$, then the three main kinds of compactness are equivalent in $X$. As corollaries, we obtain the following:

(1) In a paracompact and sequential space as well as a paracompact, weakly discretely generated and AP space, the three main kinds of compactness are equivalent.

(2) Let $X$ be a compact space and $Y$ a compact metrizable space. Then the three main kinds of compactness are equivalent in $\mathcal{C}(X, Y)([18$, Theorem 13.4.3 (The Eberlein-Smulian Theorem)]).

(3) Let $X$ be a compact space. Then the three main kinds of compactness are equivalent in $C_{p}(X)$.

\section{Results}

Consider the following property of a space $X$.

(*) For each countably compact subset $S$ of $X$, the closure $\bar{S}$ of $S$ in $X$ is compact.

It is obvious that a space $X$ satisfies $(*)$ if and only if for each countably compact subset $S$ of $X$, there exists a compact subset $K$ of $X$ such that $S \subset K$. Hence, it follows directly that every compact space satisfies $(*)$ and a countably compact and non-compact space does not satisfy $(*)$. It is also clear that every second countable space satisfies $(*)$ and hence the real line $\mathbb{R}$ with the usual topology satisfies $(*)$. A subset $S$ of $X$ is relatively compact ([6] and [18]) if and only if the closure $\bar{S}$ of $S$ in $X$ is compact. Hence a space $X$ satisfies (*) if and only if every countably compact subset of $X$ is relatively compact.

Recall that a space $X$ is called semi-metrizable ([9]) if there exists a symmetric (= a metric except for the triangle inequality) $d$ on $X$ such that for each $x \in X$, the family $\{B(x, r): r>0\}$ forms a (not necessarily open) neighborhood base at $x$, where $B(x, r)$ denotes the set $\{y \in X: d(x, y)<r\}$. A space $X$ is paracompact ([6]) if and only if each open cover of $X$ has an open neighborhood-finite refinement.

Obviously, every semi-metrizable space is a first countable and paracompact space, every compact space is paracompact and being first countable and being paracompact are independent (see [6], [9] and [12]).

First we will show some sufficient conditions for a space to satisfy $(*)$.

Theorem 2.1. Every paracompact and sequential space satisfies (*).

Proof. Let $S$ be a countably compact subspace of a paracompact and sequential space $X$. By Theorem 1.1(5), since $X$ is a sequential space and $S$ is a countably compact subspace of $X, S$ is closed in $X$. And since $X$ is a paracompact space and $S$ is closed in $X, S$ is also paracompact. Note that every paracompact and countably compact space is compact ([6, XI,Corollary 3.4]). Thus $S$ is compact and therefore $X$ satisfies $(*)$.

By Theorem 2.1, it is clear that every semi-metrizable space satisfies $(*)$. 
Still there is a very natural question left open: Does a paracompact and AP space satisfy $(*)$ ? However, we now have the following theorem.

Recall that a space $X$ is weakly discretely generated ([5]) if for each nonclosed subset $A$ of $X$, there exist $x \in \bar{A}-A$ and a subset $D$ of $A$ such that $D$ is discrete and $x \in \bar{D}$. A space $X$ is a countable tightness ([1]) (also called closure countable ([18])) space if and only if for each subset $A$ of $X$ and each $x \in \bar{A}$, there exists a countable subset $B$ of $A$ such that $x \in \bar{B}$. It is well-known that every sequential space is a weakly discretely generated and countable tightness space (see [5, Proposition 3.3(3)] and [10]).

From the following example, we see that a weakly discretely generated, countable tightness and AP space need not be sequential in general.

Example 2.2. Let $X=\{(0,0)\} \cup(\mathbb{N} \times \mathbb{N})$. We define a topology $\tau$ for $X$ by for each $(m, n) \in X-\{(0,0)\},\{(m, n)\} \in \tau$ and $(0,0) \in U \in \tau$ if and only if for all but a finite number of integers $m$, the sets $\{n \in \mathbb{N}:(m, n) \notin U\}$ are each finite. Hence each point $(m, n) \in X-\{(0,0)\}$ is isolated and each open neighborhood of $(0,0)$ contains all but a finite number of points in each of all but a finite number of columns (see Arens-Fort space in [15]). Then it is clear that the space $X$ is a Hausdorff, weakly discretely generated and countable tightness space and there is a unique non-isolated point $(0,0)$ in $X$. Note that any space with a unique non-isolated point is $\operatorname{AP}([16$, Proposition 2.1(10)]). Thus $X$ is AP. But, it is not sequential. For, $(0,0) \in \overline{\mathbb{N}} \times \mathbb{N}$, there does not exist any sequence of points of $\mathbb{N} \times \mathbb{N}$ converging to $(0,0)([15,26(3)])$.

Lemma 2.3 ([11, Theorem 2.11]). Every countably compact subset of a weakly discretely generated and AP space $X$ is closed in $X$.

Theorem 2.4. Every paracompact, weakly discretely generated and AP space $X$ satisfies $(*)$.

Proof. From Lemma 2.3, this follows in the same manner as in the proof of Theorem 2.1.

We will give some examples of spaces with $(*)$ and a space which is neither countably compact nor does it satisfy $(*)$.

Example 2.5. (1) The space of ordinals $X=\left[0, \omega_{1}\right]$, where $\omega_{1}$ denotes the first uncountable ordinal is a Hausdorff compact (and hence paracompact) space. Since $X$ is compact, $X$ satisfies (*). But, $X$ is neither sequential ([7]) nor AP ([16, Corollary 2.10]); moreover it does not have countable tightness.

(2) Let $Y=\left[0, \omega_{1}\right] \times[0, \omega]-\left\{\left(\omega_{1}, \omega\right)\right\}$, where $\omega$ denotes the first limit ordinal. Then since the set $\left\{\left(\omega_{1}, n\right): n \in \mathbb{N}\right\}$ has no cluster point in $Y, Y$ is not a countably compact space. Clearly, $\left[0, \omega_{1}\right) \times\{\omega\}$ is closed in $Y$ and it is homeomorphic to $\left[0, \omega_{1}\right)$. Since $\left[0, \omega_{1}\right)$ is a countably compact and non-compact space, we have that $Y$ does not satisfy $(*)$.

(3) Let $X$ be the set of all real numbers and let $A=\left\{\frac{1}{n}: n \in \mathbb{N}\right\}$. Define a topology $\tau$ for $X$ by letting $O \in \tau$ if $O=U-B$, where $B \subset A$ and $U$ is 
an open set in the usual topology for the real line. The topology $\tau$ is called the Smirnov topology for $X$. Then the space $X$ is Hausdorff, but it is neither regular nor paracompact (and hence not compact)(see [15, p. 86, 64]). Clearly, $X$ is secound countable. It follows that $X$ is not countably compact and it satisfies $(*)$.

(4) Let $X$ be a compact space and $Y$ a compact metrizable space. Let $\mathcal{C}(X, Y)$ be the space of all continuous functions from $X$ into $Y$ endowed with the topology of pointwise convergence; i.e., the relative topology as a subspace of $Y^{X}$ with the Tychonoff product topology. Then the space $\mathcal{C}(X, Y)$ satisfies (*) $([18$, Lemma 13.4.5]).

(5) In [1, III, Corollary 4.3], A. V. Arhangel'skii proved the following theorem called the classical statement of Grothendieck's theorem: Let $X$ be a countably compact space and let $S \subset C_{p}(X)$ be a countably compact subspace of $C_{p}(X)$. Then the closure of $S$ in $C_{p}(X)$ is compact, where $C_{p}(X)$ denotes the space of all real-valued continuous functions on $X$ endowed with the topology of pointwise convergence. Hence we know that if $X$ is a countably compact space, then $C_{p}(X)$ satisfies $(*)$.

We now study on spaces in which the three main kinds of compactness are equivalent.

Theorem 2.6. Let $X$ be a space with $(*)$. If each countably compact subspace of $X$ is a sequential space, then the three main kinds of compactness are equivalent in $X$; that is, for each subspace $S$ of $X$, the following statements are equivalent.

(1) $S$ is compact.

(2) $S$ is countably compact.

(3) $S$ is sequentially compact.

Proof. By definitions and [6, XI, Theorem 3.2], (1) $\Rightarrow(2)$ and $(3) \Rightarrow(2)$ are clear.

$(2) \Rightarrow(1)$ Let $S$ be a countably compact subspace of $X$. Then by $(*), \bar{S}$ is compact. It is sufficient to show that $S$ is closed in $X$. Since every compact space is countably compact, by hypothesis, $\bar{S}$ is a sequential space. Since $S$ is also a countably compact subspace of $\bar{S}$, by Theorem 1.1(5) we have that $S$ is closed in $\bar{S}$. Hence $S$ is closed in $X$.

$(2) \Rightarrow(3)$ Let $S$ be a countably compact subspace of $X$. Then by hypothesis, $S$ is a sequential space. Since every sequential space is WAP, by Theorem $1.1(6), S$ is sequentially compact.

Lemma 2.7. Let $X$ be a sequential space with (*). Then each countably compact subspace of $X$ is a sequential space.

Proof. Let $S$ be a countably compact subspace of $X$. Then by $(*), \bar{S}$ is compact. Since $X$ is a sequential space, by Theorem 1.1(3), $\bar{S}$ is a sequential space. Since $S$ is also a countably compact subspace of $\bar{S}$, by Theorem 1.1(5), $S$ is closed in $\bar{S}$ and thus $S$ is closed in $X$. From Theorem 1.1(3), it follows that $S$ is a sequential space. 
Remark 2.8. In [8, 7.1 Example and 7.2 Proposition], S. P. Franklin showed that a sequential and compact space need not be Fréchet-Urysohn in general and a sequential space is Fréchet-Urysohn if and only if it is hereditarily sequential. From the facts above, it follows immediately that a sequential and compact space is not hereditarily sequential. Since a compact space satisfies $(*)$, we have that a sequential space with $(*)$ is not hereditarily sequential.

Theorem 2.9. Let $X$ be a sequential space with (*). Then the three main kinds of compactness are equivalent in $X$.

Proof. This follows from Theorem 2.6 and Lemma 2.7.

Lemma 2.10. Let $X$ be a space with $(*)$. If every countably compact subspace of $X$ is an AP space, then each countably compact subspace of $X$ is closed in $X$.

Proof. Let $S$ be a countably compact subspace of $X$. Then by $(*), \bar{S}$ is compact. By hypothesis, $\bar{S}$ is an AP space. From Theorem 1.1(4), it follows that $\bar{S}$ is a Fréchet-Urysohn space. Since $S$ is also a countably compact subspace of $\bar{S}$ and since every Fréchet-Urysohn space is a sequential space, by Theorem 1.1(5), $S$ is closed in $\bar{S}$ and thus $S$ is closed in $X$.

Theorem 2.11. Let $X$ be a space with (*). If each countably compact subspace of $X$ is an AP space, then the three main kinds of compactness are equivalent in $X$.

Proof. Let $S$ be a compact or sequentially compact subspace of $X$. Then as noted in proof of Theorem 2.6, $S$ is countably compact.

Let $S$ be a countably compact subspace of $X$. From Lemma 2.10, $S$ is closed in $X$ and so $S=\bar{S}$. By $(*), \bar{S}$ is compact and hence $S$ is compact.

Let $S$ be a countably compact subspace of $X$. Then by hypothesis, $S$ is an AP space and so WAP. It follows that $S$ is sequentially compact from Theorem $1.1(6)$.

Theorem 2.12. Let $X$ be an AP space with (*). Then the three main kinds of compactness are equivalent in $X$.

Proof. This follows from Theorem 1.1(2) and Theorem 2.11.

Remark 2.13. (1) Naturally, we now have a question whether Theorem 2.9 or Theorem 2.12 does hold for any WAP space with $(*)$. In Example 2.5(1), the space of ordinals $X=\left[0, \omega_{1}\right]$ satisfies $(*)$, but it is neither sequential nor AP. It is clear that the subspace $\left[0, \omega_{1}\right)$ of $X$ is a countably compact and non-compact space. Note that $X$ is a scattered space (i.e., every non-empty subspace of $X$ has an isolated point) $[15$, p. $70,43(14)]$ and every scattered space is a WAP space $([16$, Theorem 2.7]). Hence $X$ is a WAP space. Thus we have that compactness and countable compactness are not equivalent in a WAP space with $(*)$, so the answer of this question is negative. 
(2) It is well-known that every countably compact space is pseudo-compact and in weakly normal spaces pseudo-compactness is equivalent to countable compactness $([6])$. In [15, Irrational Slope Topology], there is an example of a space that is Hausdorff, second countable and pseudo-compact, but not countably compact. Since every second countable space satisfies $(*)$, we see that a second countable (and hence sequential and AP), pseudo-compact space with $(*)$ need not be countably compact in general.

From the previous results, as corollaries, we obtain the followings:

Corollary 2.14. Let $X$ be a paracompact and sequential space. Then the three main kinds of compactness are equivalent in $X$.

Proof. This follows from Theorems 2.1 and 2.9 .

Corollary 2.15. Let $X$ be a paracompact, weakly discretely generated and $A P$ space. Then the three main kinds of compactness are equivalent in $X$.

Proof. This follows from Theorems 2.4 and 2.12 .

Corollary 2.16 ([18, Theorem 13.4.3 (The Eberlein-Smulian Theorem)]). Let $X$ be a compact space and $Y$ a compact metrizable space. Then the three main kinds of compactness are equivalent in $\mathcal{C}(X, Y)$.

Proof. In Example 2.5(4), the space $\mathcal{C}(X, Y)$ satisfies $(*)$. Let $S$ be a countably compact subspace of $\mathcal{C}(X, Y)$. Then by $(*), \bar{S}$ is compact. Note that every compact subspace of $\mathcal{C}(X, Y)$ is a Fréchet-Urysohn space ([18, Theorem 13.4.2]). Hence, $\bar{S}$ is a Fréchet-Urysohn space. By Theorem 1.1(1), $S$ is also FréchetUrysohn and so sequential and AP. Thus this follows from either of Theorems 2.6 or 2.11 .

Corollary 2.17. Let $X$ be a compact space. Then the three main kinds of compactness are equivalent in $C_{p}(X)$.

Proof. In Example 2.5(5), $C_{p}(X)$ satisfies $(*)$. Note that since $X$ is a compact space, $C_{p}(X)$ is an AP space $([4$, Theorem 3.6]). Thus this follows from Theorem 2.12.

Remark 2.18. (1) From either of Corollaries 2.14 or 2.15, we have directly a well-known theorem that the three main kinds of compactness are equivalent in a paracompact and first countable space $([12$, p. 142, H]) (and hence in a semi-metrizable space).

(2) Note that for the closed interval $[0,1]$ in the real line with the usual topology, the space $C_{p}([0,1])$ is not sequential ([1, II, Lemma 3.5]). Hence, in the proof of Corollary 2.17, the space $C_{p}(X)$ need not be a sequential space in general. Thus we know that Corollary 2.17 does not follow from Theorem 2.9.

Acknowledgement. The author wish to thank the referee for his/her careful reading of the paper. 


\section{References}

[1] A. V. Arhangel'skii, Topological Function Spaces, Mathematics and its Applications (Soviet Series), 78. Kluwer Academic Publishers Group, Dordrecht, 1992.

[2] A. V. Arhangel'skii and L. S. Pontryagin (Eds.), General Topology I, Encyclopaedia of Mathematical Sciences, vol.17, Springer-Verlage, Berlin, 1990.

[3] A. Bella, On spaces with the property of weak approximation by points, Comment. Math. Univ. Carolin. 35 (1994), no. 2, 357-360.

[4] A. Bella and I. V. Yaschenko, On AP and WAP spaces, Comment. Math. Univ. Carolin. 40 (1999), no. 3, 531-536.

[5] A. Dow, M. G. Tkachenko, V. V. Tkachuk, and R. G. Wilson, Topologies generated by discrete subspaces, Glas. Mat. Ser. III 37(57) (2002), no. 1, 187-210.

[6] J. Dugundji, Topology, Allyn and Bacon, Inc., Boston, 1970.

[7] S. P. Franklin, Spaces in which sequences suffice, Fund. Math. 57 (1965), 107-115.

[8] _ Spaces in which sequences suffice. II, Fund. Math. 61 (1967), 51-56.

[9] G. Gruenhage, Generalized metric spaces, Handbook of set-theoretic topology, 423-501, North-Holland, Amsterdam, 1984

[10] W. C. Hong, Generalized Fréchet-Urysohn spaces, J. Korean Math. Soc. 44 (2007), no. 2, 261-273.

[11] - On spaces in which compact-like sets are closed, and related spaces, Commun. Korean Math. Soc. 22 (2007), no. 2, 297-303.

[12] J.-I. Nagata, Modern General Topology, North-Holland Publishing Co., AmsterdamLondon, 1974.

[13] F. Obersnel, Some notes on weakly Whyburn spaces, Topology Appl. 128 (2003), no. 2-3, 257-262.

[14] J. Pelant, M. G. Tkachenko, V. V. Tkachuk, and R. G. Wilson, Pseudocompact Whyburn spaces need not be Fréchet, Proc. Amer. Math. Soc. 131 (2003), no. 10, 3257-3265.

[15] L. A. Steen and J. A. Seebach, Jr., Counterexamples in Topology, Springer-Verlag, New York-Heidelberg, 1978.

[16] V. V. Tkachuk and I. V. Yaschenko, Almost closed sets and topologies they determine, Comment. Math. Univ. Carolin. 42 (2001), no. 2, 395-405.

[17] J. E. Vaughan, Countably compact and sequentially compact spaces, Handbook of settheoretic topology, 569-602, North-Holland, Amsterdam, 1984.

[18] A. Wilansky, Topology for Analysis, Waltham, Mass.-Toronto, Ont.-London, 1970.

Department of Mathematics Education

Pusan National University

PusAn 609-735, Korea

E-mail address: wchong@pusan.ac.kr 